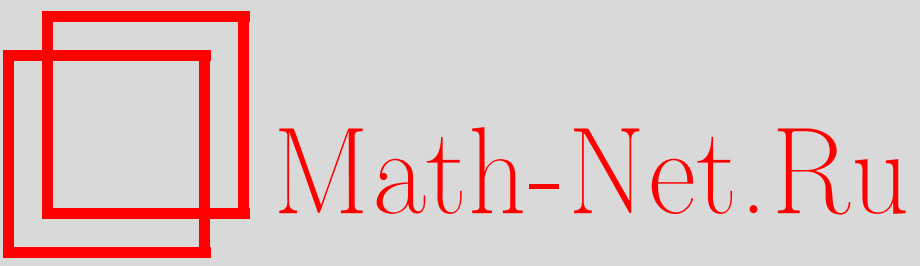

А. А. Джалилов, К. М. Ханин, Об инвариантной мере для гомеоморфизмов окружности с одной точкой излома, УМН, 1996, том 51, выпуск 6, 201-202

DOI: https://doi.org/10.4213/rm1027

Использование Общероссийского математического портала Math-Net.Ru подразумевает, что вы прочитали и согласны с пользовательским соглашением

http://www.mathnet.ru/rus/agreement

Параметры загрузки:

IP: 54.237 .206 .68

26 апреля 2023 г., 13:39:26 


\title{
ОБ ИНВАРИАНТНОЙ МЕРЕ ДЛЯ ГОМЕОМОРФИЗМОВ ОКРУЖНОСТИ С ОДНОЙ ТОЧКОЙ ИЗЛОМА
}

\author{
А. А. ДжАлилов, К. М. ХАнин
}

В настоящей работе изучаются гомеоморфизмы окружности, задаваемые функциями с одной точкой излома, в которой происходит скачок первой производной. А именно, пусть $f(x)$ непрерывная строго монотонная функция такая, что

a) $f(x+1)=f(x)+1$;

б) существует точка $x_{c} \in S^{1}=[0,1)$, для которой $\sqrt{f^{\prime}\left(x_{c}-0\right) / f^{\prime}\left(x_{c}+0\right)}=c \neq 1$;

c) $f(x) \in C^{2+\nu}\left(S^{1} \backslash\left\{x_{c}\right\}, f^{\prime}(x) \geqslant\right.$ const $>0, x \in S^{1} \backslash\left\{x_{c}\right\}$.

Определим гомеоморфизм окружности $T$, отвечающий $f: T x=\{f(x)\}, x \in S^{1}$, где $\{\cdot\}$ обозначает дробную часть числа. Обозначим $\rho=\rho(T)$ соответствующее число вращения для $T[1]$. Предположим, что $\rho$ иррационально. Тогда отображение $T$ является строго эргодическим [1]. Единственную инвариантную меру обозначим через $\mu$.

Теорема 1. Инвариантная мера $\mu$ гомеоморфизма Т сингулярна относительно мерыл Лебега $\lambda$.

При доказателстве теоремы 1 мы используем обшие свойства динамических разбиений и двойные отношения (cross-ratio).

Положим $\varphi=\mu([0, x]), x \in S^{1}$. Хорошо известно [1], [2], что отбражение $\varphi$ сопрягает $T$ с линейным поворотом $T_{\rho} x=\{x+\rho\}, x \in S^{1}$, т.е. имеет место равенство $\varphi(T x)=T_{\rho} \varphi(x)$, $x \in S^{1}$.

Пусть имеется четверка чисел $(a, b, c, d), a, b, c, d$. Введем двойные отношения (cross-ratio), отвечающие этой четверке, по формуле $\operatorname{Cr}(a, b, c, d)=\frac{(b-a)(d-c)}{(c-a)(d-b)}$. Свитек в [3] интенсивно использовал двойные отношения для анализа гомеоморфизмов окружности.

Сформулируем основные леммы и, используя их, докажем теорему 1.

Лемма 1. Пусть $\varphi^{\prime}\left(x_{0}\right)=\alpha>0, x_{0} \in S^{1}$. Тогда для любого $\varepsilon>0$ существует $\delta=$ $\delta\left(x_{0}, \varepsilon\right)>0$, при котором для любой четверки точек $\left(z_{1}, z_{2}, z_{3}, z_{4}\right), z_{1}<z_{2}<z_{3}<z_{4}$, такой, что

a) $z_{i} \in\left(x_{0}-\delta, x_{0}+\delta\right)$

b) пусть $a=z_{2}-z_{1}, b=z_{3}-z_{2}, c=z_{4}-z_{3}$, тогда для некоторой константы $R>1 \quad R^{-1} a \leqslant b \leqslant R a, R^{-1} a \leqslant c \leqslant R a$

c) $\max \left(\left|z_{1}-x_{0}\right|,\left|z_{2}-x_{0}\right|,\left|z_{3}-x_{0}\right|,\left|z_{4}-x_{0}\right|\right) \leqslant R a$;

выполнено неравенство

$$
\left|\frac{\operatorname{Cr}\left(\varphi\left(z_{1}\right), \varphi\left(z_{2}\right), \varphi\left(z_{3}\right), \varphi\left(z_{4}\right)\right)}{\operatorname{Cr}\left(z_{1}, z_{2}, z_{3}, z_{4}\right)}-1\right|<c_{1} \varepsilon
$$

где константа $c_{1}>0$ не зависит от $\varepsilon$.

ОпредЕлЕниЕ 2. Мы скажем, что тройка отрезков $\left(\left[z_{1}, z_{2}\right],\left[z_{2}, z_{3}\right],\left[z_{3}, z_{4}\right]\right)$ покрывает особую точку $x=x_{c}$ "правилным" образом с константой $M$, если

1) $x_{c} \in\left[z_{1}, z_{2}\right]$ (или $\left.x_{c} \in\left[z_{3}, z_{4}\right]\right)$ и $x_{c} \notin\left[z_{2}, z_{3}\right]$;

2) $\left(x_{c}-z_{1}\right) /\left(z_{2}-z_{1}\right) \geqslant M$ (или $\left.\left(z_{4}-x_{c}\right) /\left(z_{4}-z_{3}\right) \geqslant M\right)$.

ЛЕмма 2. Пусть определяющая функция $f(x)$ гомеоморфизма Т удовлетворяет условиям теоремь 1 для некоторой точки $x_{0}$ и сопряжсене $\varphi$ дифференцируемо, т.е. $\varphi^{\prime}\left(x_{0}\right)=\alpha>0, x_{0} \in S^{1}, x_{0} \notin\left\{T^{(i)} x_{c}, i \in Z^{1}\right\}$. Тогда для бесконечного числа натуральных $n$ существуют отрезки $\left[z_{i}, z_{i+1}\right] \in\left(x_{0}-\delta, x_{0}+\delta\right), i=1,2,3$, и константа $M$, $0<M<1$, со следующими свойствами:

1) $\left|T^{(i)}\left[z_{1}, z_{4}\right]\right| \leqslant \operatorname{const} \beta^{n}, 0 \leqslant i \leqslant q_{n}$, əде $\beta=\left(1+e^{v}\right)^{-1 / 2}<1, v=\operatorname{var}_{S^{1}} \ln f^{\prime}(x)$, $\cdot \cdot \mid$ означает длину отрезка;

2) $\sum_{i=0}^{q_{n}}\left|T^{(i)}\left[z_{1}, z_{4}\right]\right| \leqslant K$, әде константа $K>0$ не зависит от $n u \varepsilon$; 
3) отрезки $\left[z_{i}, z_{i+1}\right], i=1,2,3, u\left[T^{q_{n}} z_{i}, T^{q_{n}} z_{i+1}\right], i=1,2,3$, удовлетворяют условиям леммы 1 ;

4) система из тройки отрезков ([T $\left.\left.{ }^{i} z_{1}, T^{i} z_{2}\right],\left[T^{i} z_{2}, T^{i} z_{3}\right],\left[T^{i} z_{3}, T^{i} z_{4}\right]\right), 0 \leqslant i \leqslant q_{n}$, покрывает особую точку $x=x_{c}$ не более чем два раза, причем хотя бы одна тройка отрезков покрывает ее "правильным" образом с константой $M$; кроме moro, $x_{c} \notin \bigcup_{i=0}^{q_{n}}\left[T^{i}\left[z_{2}, z_{3}\right]\right]$.

Лемма 3. Предположим, что отрезки $\left[z_{i}, z_{i+1}\right], i=1,2,3$, удовлетворяют утверждениям 1)-4) леммы 2. Тогда для достоточно больщих натуральных $n$ имеют место следующие неравенства:

a) рри с $>1 \frac{\operatorname{Cr}\left(T^{q_{n}} z_{1}, T^{q_{n}} z_{2}, T^{q_{n}} z_{3}, T^{q_{n}} z_{4}\right)}{\operatorname{Cr}\left(z_{1}, z_{2}, z_{3}, z_{4}\right)}>$ const $>1$;
b) $n$; $~ c<1 \frac{\operatorname{Cr}\left(T^{q_{n}} z_{1}, T^{q_{n}} z_{2}, T^{q_{n}} z_{3}, T^{q_{n}} z_{4}\right)}{\operatorname{Cr}\left(z_{1}, z_{2}, z_{3}, z_{4}\right)}<$ const $<1$.

Докажем теперь теорему 1 . По определению $\varphi(x)=\int_{S^{1}} d \mu(x), \quad x_{0} \in S^{1}$. Отсюда видно, что $\varphi(x)$ является неубывающей функцией. По теореме Лебега о дифференцируемости монотонной функции (см. [4, с. 324]) на множестве “полной" мере (по мере Лебега) функция $\varphi(x)$ имеет конечные производные $\varphi^{\prime}(x)$. Это множество обозначим через $X$. Поскольку мера Лебега $\lambda$ и инвариантная мера $\mu$ являются непрерывными мерами имеем: $\lambda\left\{T^{(i)}\left(x_{c}\right), i \in Z^{1}\right\}=$ $\mu\left\{T^{(i)}\left(x_{c}\right), i \in Z^{1}\right\}=0$. Обозначим $X_{1}=X\left\{T^{(i)} x_{c}, i \in Z^{1}\right\}$. Очевидно, что $\lambda(X)=\mu(X)=1$. Для доказательства теоремы 1 нам достоточно показать, что $\varphi^{\prime}(x) \equiv 0$ на множестве $X_{1}$.

Предположим, что $\varphi^{\prime}(x)=\alpha>0, x_{0} \in X_{1}$. Для определенности предположим, что величина излома $c>1$. Фиксируем $\varepsilon>0$. Возьмем тройку отрезков $\left(\left[z_{1}, z_{2}\right],\left[z_{2}, z_{3}\right],\left[z_{3}, z_{4}\right]\right),\left[z_{i}, z_{i+1}\right] \in$ $\left(x_{0}-\delta, x_{0}+\delta\right), i=\overline{1,3}$, удовлетворяюшие условиям леммы 3 . В силу леммы 1 :

$$
\begin{gathered}
\left|\frac{\operatorname{Cr}\left(\varphi\left(z_{1}\right), \varphi\left(z_{2}\right), \varphi\left(z_{3}\right), \varphi\left(z_{4}\right)\right)}{\operatorname{Cr}\left(z_{1}, z_{2}, z_{3}, z_{4}\right)}-1\right|<c_{1} \varepsilon, \\
\left|\frac{\operatorname{Cr}\left(\varphi\left(T^{\left(q_{n}\right)} z_{1}\right), \varphi\left(T^{\left(q_{n}\right)} z_{2}\right), \varphi\left(T^{\left(q_{n}\right)} z_{3}\right), \varphi\left(T^{\left(q_{n}\right)} z_{4}\right)\right)}{\operatorname{Cr}\left(T^{\left(q_{n}\right)} z_{1}, T^{\left(q_{n}\right)} z_{2}, T^{\left(q_{n}\right)} z_{3}, T^{\left(q_{n}\right)} z_{4}\right)}-1\right|<c_{1} \varepsilon .
\end{gathered}
$$

Используя уравнение для сопряжения $\varphi(T x)=T \rho \varphi(x)$ легко можно показать, что (3) $\operatorname{Cr}\left(\varphi\left(T^{\left(q_{n}\right)} z_{1}\right), \varphi\left(T^{\left(q_{n}\right)} z_{2}\right), \varphi\left(T^{\left(q_{n}\right)} z_{3}\right), \varphi\left(T^{\left(q_{n}\right)} z_{4}\right)\right)=\operatorname{Cr}\left(\varphi\left(z_{1}\right), \varphi\left(z_{2}\right), \varphi\left(z_{3}\right), \varphi\left(z_{4}\right)\right)$.

Из (1)-(3) непосредственно вытекает, что

$$
\left|\frac{\operatorname{Cr}\left(T^{\left(q_{n}\right)} z_{1}, T^{\left(q_{n}\right)} z_{2}, T^{\left(q_{n}\right)} z_{3}, T^{\left(q_{n}\right)} z_{4}\right)}{\operatorname{Cr}\left(z_{1}, z_{2}, z_{3}, z_{4}\right)}-1\right|<c_{3} \varepsilon,
$$

где константа $c_{3}$ не зависит от $\varepsilon$ и $n$.

С другой стороны, из леммы 3 следует, что для бесконечного числа натуральных $n$

$$
\frac{\operatorname{Cr}\left(T^{\left(q_{n}\right)} z_{1}, T^{\left(q_{n}\right)} z_{2}, T^{\left(q_{n}\right)} z_{3}, T^{\left(q_{n}\right)} z_{4}\right)}{\operatorname{Cr}\left(z_{1}, z_{2}, z_{3}, z_{4}\right)}>\text { const }>1 .
$$

При достаточно малых $\varepsilon$, последнее неравенство и неравенство (4) одновременно не могут выполнятся. Это противоречие доказывает теорему 1.

\section{СПИСОК ЛИТЕРАТУРЫ}

[1] Корнфельд И. П., Синай Я.Г., Фомин С. В. Эргодическая теория. М.: Наука, 1980. [2] Khanin K. M., Vul E. V. // Adv. Soviet Math. 1991. V. 3. P. 57-98. [3] Swiatek G. // Comm. Math. Phys. 1988. V. 119. № 2. P. 109-128. [4] Колмогоров А. Н., Фомин С. В. Элементы теории функции и функционального анализа. М.: Наука, 1976.

Самаркандский государственный университет, Heriot-Watt University, Edinburgh 